\section{Blue-White Selection Step Enhances the Yield of SAGE Concatemers}

\section{BioTechniques 32:484-486 (March 2002)}

In recent years, serial analysis of gene expression (SAGE) has been widely employed to examine mRNA transcription profiles of various cell lines and tissues $(6,8,10-12)$. SAGE is based on the generation of a unique 10-11-bp tag following the most $3^{\prime}$ CATG of each mRNA transcript in the tissue or cell sample. The tags are then linked together to form long concatemers and amplified in bacteria. A random sampling of bacterial colonies is selected for sequencing, and the number of times a particular tag sequence arises provides a measure of the relative abundance of the transcript in the mRNA population. Although theoretically simple, preparation of SAGE libraries through the multiple-step protocol (10) can be difficult. Accordingly, numerous modifications have been made to the original protocol to improve the yield of constructed SAGE libraries $(1,5,7,9)$. During the successful completion and sequencing of five different SAGE libraries from rat pheochromocytoma cells [(2) and unpublished findings], we found that $13 \%-50 \%$ of the bacterial colonies selected for sequencing contained no inserts. In addition, a number of colonies contained small concatemer inserts (less than 15 tags). This significantly lengthened the time required to sequence our SAGE libraries.

Here, we describe a simple improvement in the SAGE protocol that favors the selection of bacterial colonies containing larger concatemers for DNA sequencing and that greatly reduces the number of colonies selected that do not contain concatemer inserts. This modification can be used for all versions of the SAGE method that use the pZErO'M Vector System (Invitrogen, Carlsbad, CA, USA) and substantially increases the sequencing throughput of SAGE tags.

The two main features of the pZErO vector are a Zeocin cDNA insert that provides resistance to the antibiotic

Table 1. Comparison of Tag Numbers Recovered from White and Blue Colonies

\begin{tabular}{|c|c|c|c|}
\hline & White & Blue & $\begin{array}{l}\text { Blue (Not including } \\
\text { Empty Vectors) }\end{array}$ \\
\hline Number of Empty Vectors & $0 / 38$ & \multicolumn{2}{|l|}{$5 / 38$} \\
\hline $\begin{array}{l}\text { Average Size of } \\
\text { Concatemer (bp) }\end{array}$ & \multirow[t]{2}{*}{$423 \pm 37$} & \multirow[t]{2}{*}{$264 \pm 28$} & \multirow[t]{2}{*}{$304 \pm 25$} \\
\hline Average No. & & & \\
\hline $\begin{array}{l}\text { Tags/Concatemer } \\
\text { The base pair size of the } \\
1.5 \% \text { agarose gel. For cal } \\
\text { Concatemer sizes from } 38 \\
\text { reported as averages } \pm \text { SEl }\end{array}$ & $\begin{array}{l}\quad 28 \pm 2 \\
\text { oncatemer } \\
\text { ulations, th } \\
\text { white and } 3\end{array}$ & $\begin{array}{l}18 \pm 2 \\
\text { etermined } \\
\text { vector }(2 \\
\text { colonies w }\end{array}$ & $\begin{array}{l}20 \pm 2 \\
y \text { electrophoresis in a } \\
6 \text { bp) was subtracted. } \\
\text { re determined and are }\end{array}$ \\
\hline
\end{tabular}

Zeocin and a LacZ $\alpha$-ccdB fusion cDNA insert that, when expressed, kills vector-containing bacteria by inhibiting DNA gyrase activity $(3,4)$. The insertion of cDNA into the multiple cloning site of the $\mathrm{pZErO}$ vector disrupts the inframe induction of $\mathrm{LacZ} \alpha-\mathrm{ccdB}$ cDNA expression, thereby permitting the growth of bacterial colonies. However, we and others (Chang and Moore, personal communication, Columbia University) have found while analyzing our SAGE libraries that the pZErO vector can in some instances re-ligate without inserts and allow for subsequent growth of bacterial colonies even in the presence of the Lac promoter activator IPTG. We hypothesized that such growth can occur either because a subpopulation of the DH10B bacteria is resistant to ccdB or because religation of the empty vector results in a frameshift that abolishes expression of the LacZ $\alpha$ $\operatorname{ccdB}$ fusion protein. To distinguish between these two possibilities, concatemers were first subcloned into $\mathrm{pZErO}$ as directed in step 13 of the SAGE manual (version 1.0e; www.sagenet. org), and the ligation mixture was transformed into DH10B bacteria. However, rather than growing the bacteria on LB/Zeocin agar plates as usual, the plates were first treated with 40 $\mu \mathrm{g} / \mathrm{mL} X-G a l$ and $1 \mathrm{mM}$ IPTG. We reasoned that if a frame shift occurred during the ligation, then colonies without inserts would be white because of the absence of LacZ $\alpha$-ccdB expression. In contrast, if ligation were in frame, then bacterial colonies without inserts would be blue because of expression of the LacZ $\alpha$-ccdB fusion protein. For the library analyzed in Table 1, analysis of both white and blue colonies revealed that empty vectors were recovered only from blue colonies. This indicates that a subpopulation of the bacteria is resistant to the levels of LacZ $\alpha$-ccdB expression achieved in our cultures. Such resistance was apparently not due to genetic drift or adaptation of the bacteria in our laboratory because colonies with insert-free vector were recovered with at least three independent batches of bacteria received directly from the supplier (Invitrogen). These findings indicate that blue-white selection can eliminate or substantially decrease the prevalence of empty vectors encountered during the sequencing steps of the SAGE protocol.

Analysis of white and blue colonies revealed that aside from empty vectors, many of the blue colonies also contained inserts (Table 1). Moreover, the presence of blue colonies was not uncommon. When we examined 392 random colonies, $54 \%$ were blue. A comparison of inserts present in blue and white colonies revealed an important difference, namely that the white colonies contained on average larger concatemers (Table 1). Analysis of concatemers from 38 white and 38 blue colonies showed that the average number of tags per concatemer is typically more than $50 \%$ greater in the white colonies than in blue colonies when colonies with empty vectors are considered in the calculation, and $40 \%$ higher when empty vectors are excluded. The reason for the difference in size of in- 
serts in blue and white colonies is not completely clear, but one possibility is that the $\mathrm{pZErO}$ vector permits expression of LacZ $\alpha$-ccdB at non-lethal levels even with insertion of foreign DNA and that this "leakiness" is reduced with longer inserts.

To further verify that blue-white selection provides a higher yield of tags per concatemers, we analyzed data from an independent library in which concatemers from random colonies were sequenced with or without bluewhite selection. For the 323 concatemers that were sequenced without prior blue-white selection, the number of recovered tags per concatemer averaged 3.9. In contrast, the yield rose by 3.5fold to an average of 13.5 tags per concatemer for 328 concatemers that had been subjected to blue-white selection.

In summary, we find that the addition of X-Gal and IPTG to bacterial plates and the use of blue-white selection significantly enhance the yield of SAGE concatemers following transfection of DH10B bacteria with pZErO vector. This avoids the need for the more time-consuming prescreening of colonies by agarose gel electrophoresis and results in a substantial enhancement in the efficiency with which SAGE concatemer libraries can be sequenced.

\section{REFERENCES}

1.Angelastro, J.M., L.P. Klimaschewski, and O.V. Vitolo. 2000. Improved NlaIII digestion of PAGE-purified $102 \mathrm{bp}$ ditags by addition of a single purification step in both the SAGE and microSAGE protocols. Nucleic Acids Res. 28:E62.

2.Angelastro, J.M., L. Klimaschewski, S. Tang, O.V. Vitolo, T.A. Weissman, L.T. Donlin, M.L. Shelanski, and L.A. Greene. 2000. Identification of diverse nerve growth factor-regulated genes by serial analysis of gene expression (SAGE) profiling. Proc. Natl. Acad. Sci. USA 97:10424-10429.

3.Couturier, M., M. el Bahassi, and L. Van Melderen. 1998. Bacterial death by DNA gyrase poisoning. Trends Microbiol. 6:269-675.

4.Hooper, D.C. 1998. Bacterial topoisomerases, anti-topoisomerases, and anti-topoisomerase resistance. Clin. Infect. Dis. 27Suppll:S54S63.

5.Kenzelmann, M. and K. Muhlemann. 1999. Substantially enhanced cloning efficiency of SAGE (serial analysis of gene expression) by adding a heating step to the original protocol. Nucleic Acids Res. 27:917-918.

6.Polyak, K. and G.J. Riggins. 2001. Gene discovery using the serial analysis of gene ex- pression technique: implications for cancer research. J. Clin. Oncol. 19:2948-2958.

7.Powell, J. 1998. Enhanced concatemer cloning-a modification to the SAGE (serial analysis of gene expression) technique. $\mathrm{Nu}-$ cleic Acids Res. 26:3445-3446.

8.Riggins, G.J. and R.L. Strausberg. 2001. Genome and genetic resources from the Cancer Genome Anatomy Project. Hum. Mol. Genet. 10:663-667.

9.St. Croix, B., C. Rago, V. Velculescu, G. Traverso, K.E. Romans, E. Montgomery, A. Lal, G.J. Riggins et al. 2000. Genes expressed in human tumor endothelium. Science 289:1197-1202.

10.Velculescu, V.E., L. Zhang, B. Vogelstein, and K.W. Kinzler. 1995. Serial analysis of gene expression. Science 270:484-487.

11.Velculescu, V.E., L. Zhang, W. Zhou, J. Vogelstein, M.A. Basrai, D.E. Bassett, Jr., P. Hieter, B. Vogelstein, and K.W. Kinzler. 1997. Characterization of the yeast transcriptome. Cell 88:243-251.

12.Velculescu, V.E., B. Vogelstein, and K.W. Kinzler. 2000. Analysing uncharted transcriptomes with SAGE. Trends Genet. 16:423-425.

We thank Claudine Bitel for her excellent technical assistance. This work is supported in part by National Institutes of Health grant nos. NS 16036 and NS33689 and the Blanchette Rockefeller Foundation to L.A.G. Address correspondence to Dr. James M. Angelastro, Dept. of Pathology, Columbia University College of Physicians and Surgeons Room Number 15-401, Center for Neurobiology and Behavior, 630 West 168th Street, New York, NY 10032, USA.e-mail:jma14@columbia.edu

Received 3 October 2001; accepted 28 November 2001.

J.M. Angelastro, E.J. Ryu, B. Törõcsik, B.K. Fiske, and L.A. Greene

Columbia University

College of Physicians and

Surgeons

New York, NY, USA

For reprints of this or any other article, contact Reprints@BioTechniques.com

\section{Rapid and Efficient Method for Suspending Cells for Neurotransmit- ter Uptake Assays}

BioTechniques 32:486-492 (March 2002)

Platelets are a convenient source of serotonin uptake transporters (SERTs) for studying the biochemical and pharmacological characteristics of serotonin transport across the plasma membrane (10). Serotonin accumulation by platelets depends on extracellular $\mathrm{Na}^{+}$ and $\mathrm{Cl}^{-}$and is inhibited by cold temperature and drugs such as fluoxetine and cocaine $(7,9,12)$. Molecular cloning has revealed that the amino acid sequence of the human platelet SERT protein is identical to the neuronal SERT (8), making the platelet a good model for neuronal serotonin uptake and a useful material in which to study drugs that modify serotonin homeostasis. We have previously reported using human platelets to study psychoactive drugs that act at the SERT (2-4). Because of known differences in the efficacy and potency of drugs acting at the human SERT compared to the SERT obtained from other species such as rodents or insects, data obtained in the human platelet system are more relevant to human pharmacology.

Human platelets can be obtained fresh or, more commonly, as outdated blood bank donor platelets. While outdated platelets may not be equivalent in all respects to fresh platelets, they do retain the ability to accumulate serotonin in a temperature- and drug-sensitive manner $(4,13,14)$. In a typical procedure, after pooling the platelets from several donors, the cells are pelleted by centrifugation. The $\mathrm{P} 1$ pellet is washed several times by floating the pellet in a balanced electrolyte buffer, followed by centrifugation. The final pellet is resuspended by manual pipetting. Manual pipetting involves the aspiration and ejection of buffer and cell pellet fragments through a pipet tip $(11,15)$; this process is repeated as many times as needed to obtain a uniform suspension. The shear forces resulting from the movement of liquid through the pipet tip cause the cell pellet to break up into 